\title{
28 Research Square \\ Clinical Characteristics and Outcomes of Rare Fungal Keratitis Caused by Verticillium dahliae
}

\author{
Qing Huang \\ Shandong Eye Hospital \\ Wenlu Zhang \\ Shandong Eye Hospital \\ Yu Sun \\ Shandong Eye Hospital \\ Xiaofeng Li \\ Shandong Eye Hospital \\ Xiaoyu Zhang \\ Shandong Eye Hospital \\ Xiuhai Lu \\ Shandong Eye Hospital \\ Xiaolin Qi ( $\nabla$ qinglianqxl@163.com ) \\ Shandong Eye Hospital
}

\section{Research Article}

Keywords: Fungal keratitis, Verticillium dahliae, In vitro antifungal susceptibility test, DNA sequence

Posted Date: March 1st, 2021

DOl: https://doi.org/10.21203/rs.3.rs-260137/v1

License: (c) (1) This work is licensed under a Creative Commons Attribution 4.0 International License.

Read Full License

Version of Record: A version of this preprint was published at Mycopathologia on November 17th, 2021. See the published version at https://doi.org/10.1007/s11046-021-00604-7. 


\section{Abstract}

\section{Purpose}

To observe clinical characteristics and treatment outcomes of fungal keratitis cause by Verticillium dahliae.

\section{Methods}

Clinical data of 7 patients diagnosed as fungal keratitis cause by V. dahliae were retrospectively analyzed. The clinical manifestations, mycology, in vitro antifungal susceptibility, treatment regimens and prognoses of the patients were evaluated.

Results

All 7 patients were farm worker, of which 5 cases were caused by plant trauma. The corneal ulcer had a round shape and a relatively limited range with the diameters mainly in the range of 2-7 $\mathrm{mm}$. The stromal infiltration was mild, and had no pseudopodia, mossiness or endothelial plaques. Intact hyphaes were detected in corneal scrapings and confocal microscopy, isolates were identified by morphology and by sequencing the internal transcribed spacer region of ribosomal DNA. In vitro antifungal susceptibility testing showed that the most sensitive antifungal drug was Amphotericin B. In the 6 patients with an ulcer less than $2 / 3$ of the corneal thickness, the ulcer healed after 18 days of antifungal treatment only in one eye. The other five patients underwent corneal ulcer debridement or conjunctival flap covering surgery. The remaining one patient with ulcer depth more than $2 / 3$ of the corneal thickness underwent lamellar keratoplasty.

\section{Conclusion}

Fungal keratitis caused by $\mathrm{V}$. dahliae has typical signs of a mild inflammatory response, and is not sensitive to antifungal drugs. It is recommended that patients undergo corneal ulcer debridement as soon as possible to promote rapid healing of the ulcers.

\section{Full Text}

Due to technical limitations, full-text HTML conversion of this manuscript could not be completed. However, the manuscript can be downloaded and accessed as a PDF.

\section{Figures}



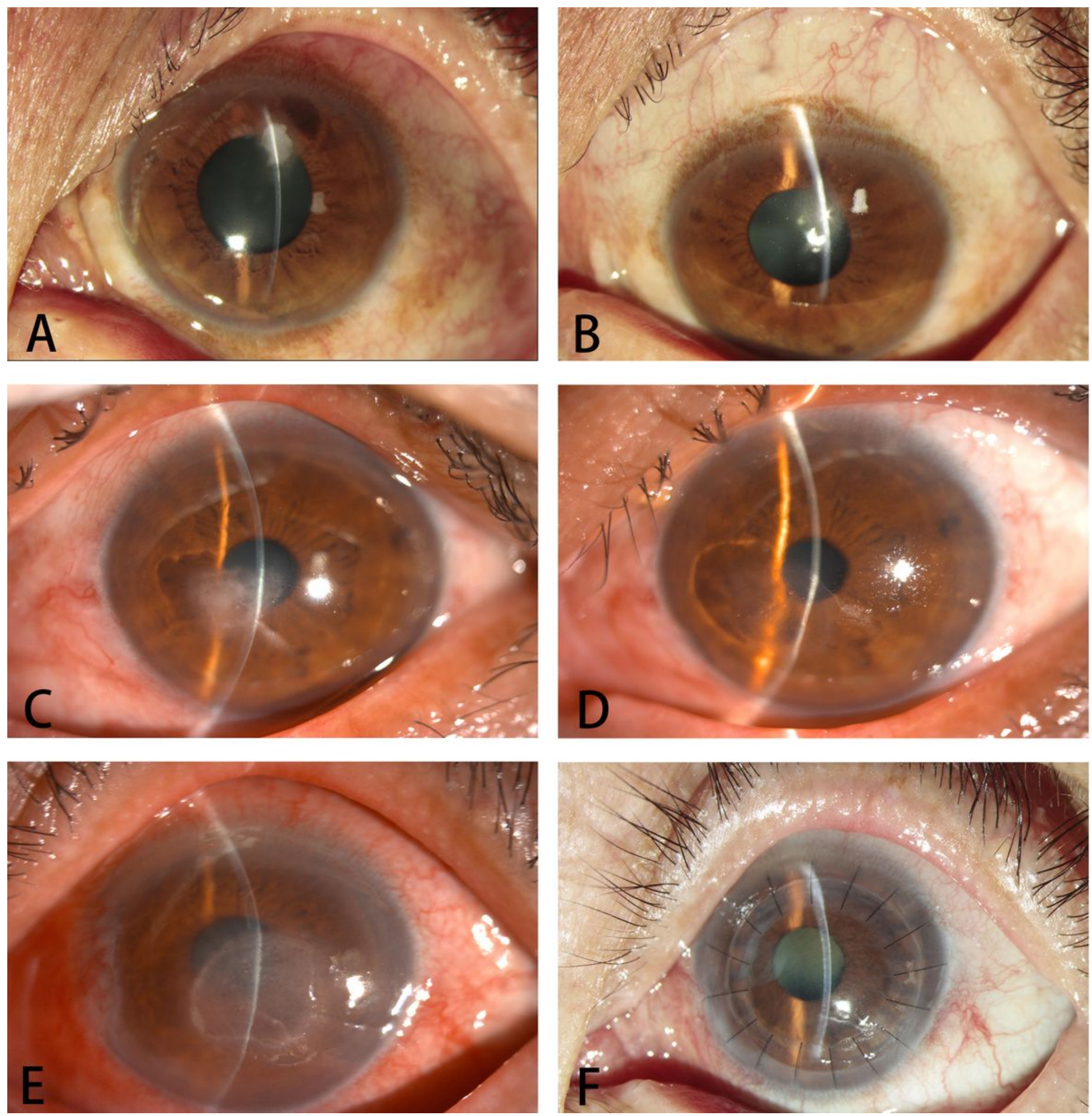

\section{Figure 1}

A Slit-lamp examination of the left eye showing a $1.5 \times 1.5 \mathrm{~mm} 2$ gray-white corneal ulcer with the depth $\leq 1 / 3$ corneal thickness, $B$ the ulcer healed after antifungal drug therapy. C Slit-lamp examination of the left eye showing a $3 \times 3 \mathrm{~mm} 2$ gray-white corneal ulcer with the depth $\leq 1 / 3$ corneal thickness, $D$ the ulcer healed after surgical debridement. e Slit-lamp examination of the left eye showing a $6 \times 7 \mathrm{~mm} 2$ gray- 
white corneal ulcer with the depth up to $2 / 3$ corneal thickness, $f$ lamellar keratoplasty was performed after ineffective antifungal drug therapy.
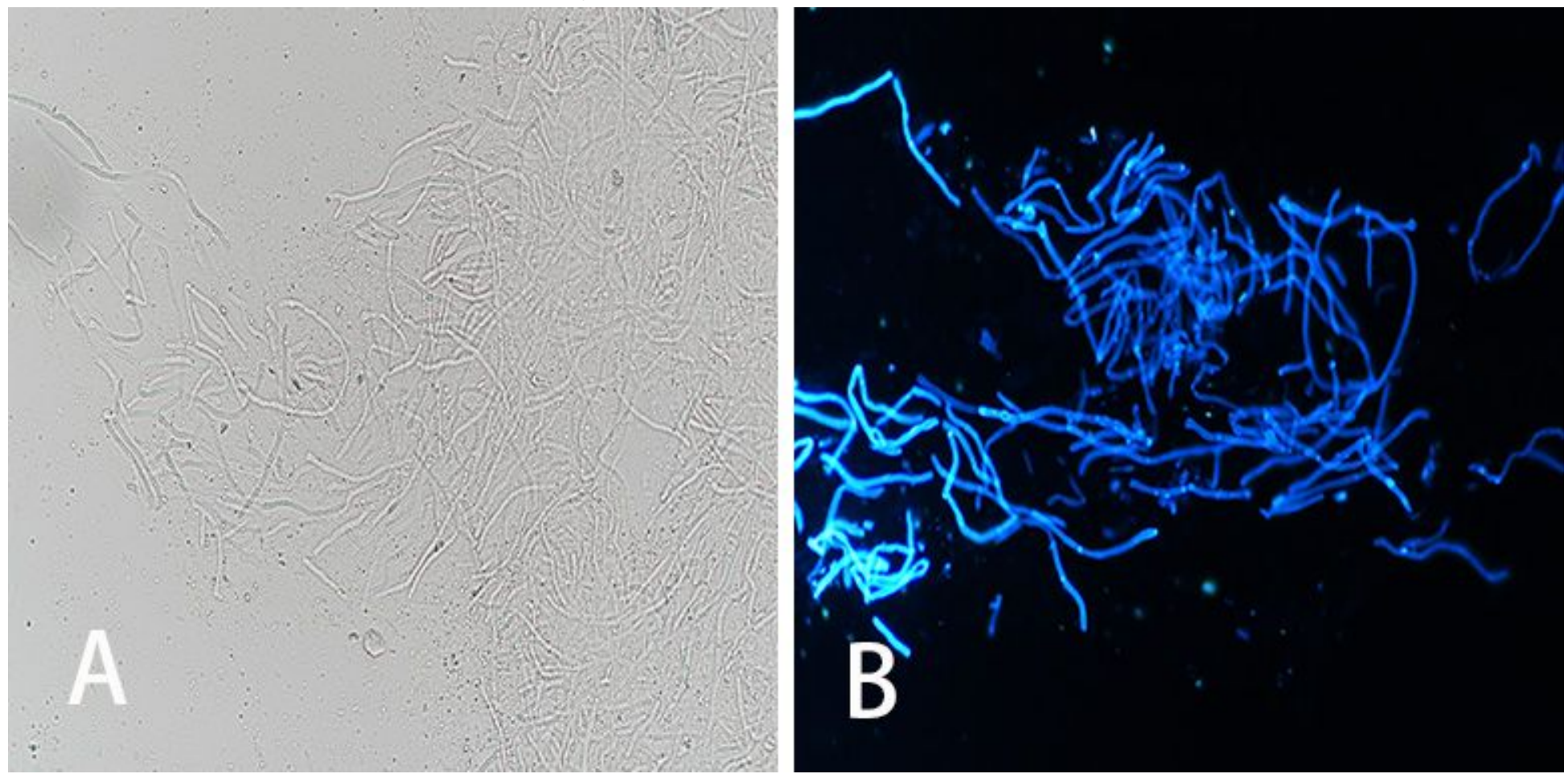

Figure 2

A $10 \%$ potassium hydroxide smear (magnification of $\times 400$ ), and B Calcofluor White Staining (magnification of $\times 400$ ) showed a large number of complete hyphae, which were thin and regular in width.
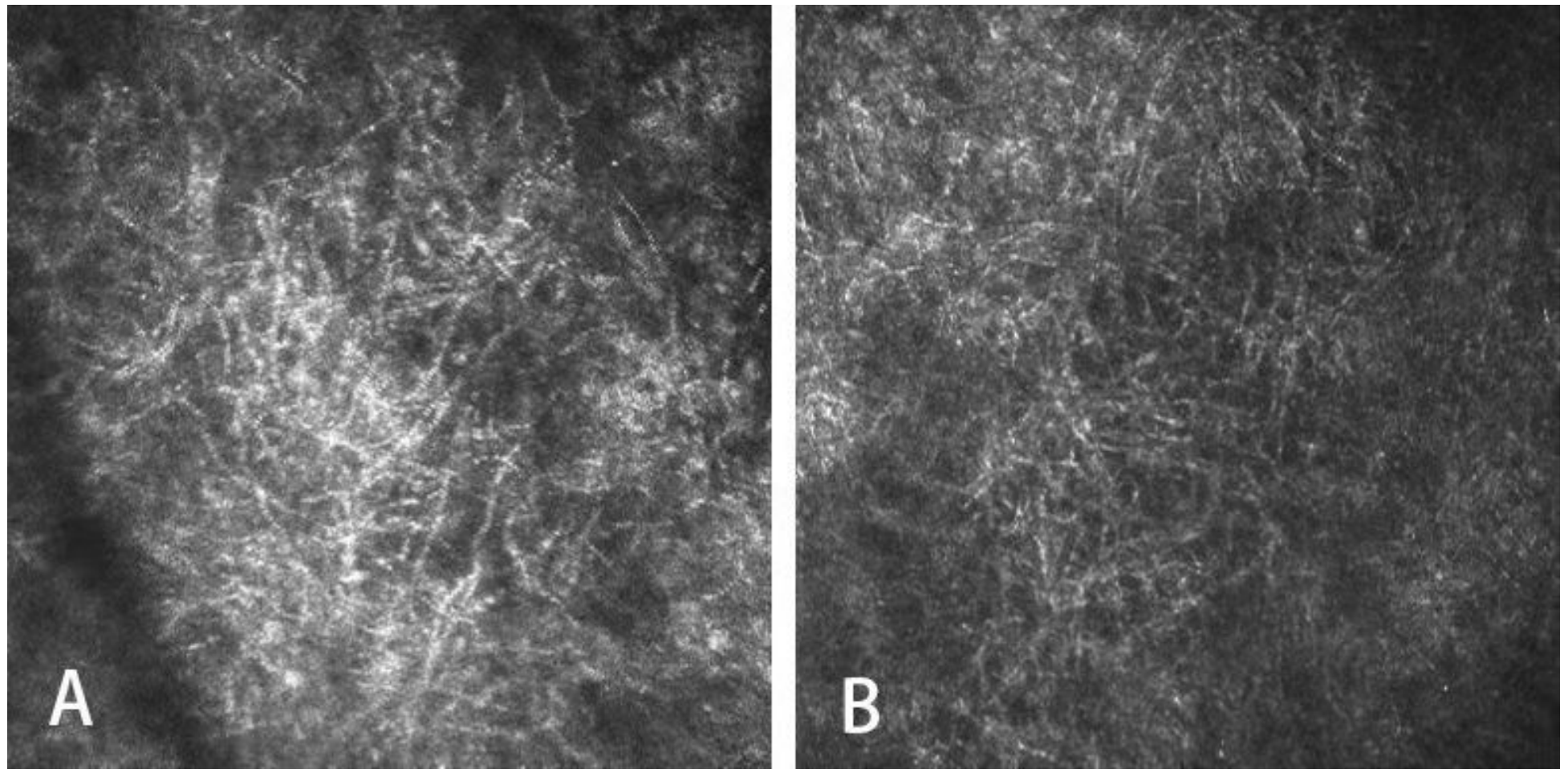

Figure 3 
A and B confocal microscopy showed a large number of hyphae were densely distributed. The hyphae were moderately reflective, upright, and slender. Their diameters were mainly 2-3 $\mu \mathrm{m}$, there were many branches, and the angles between the hyphae were small, mostly acute.
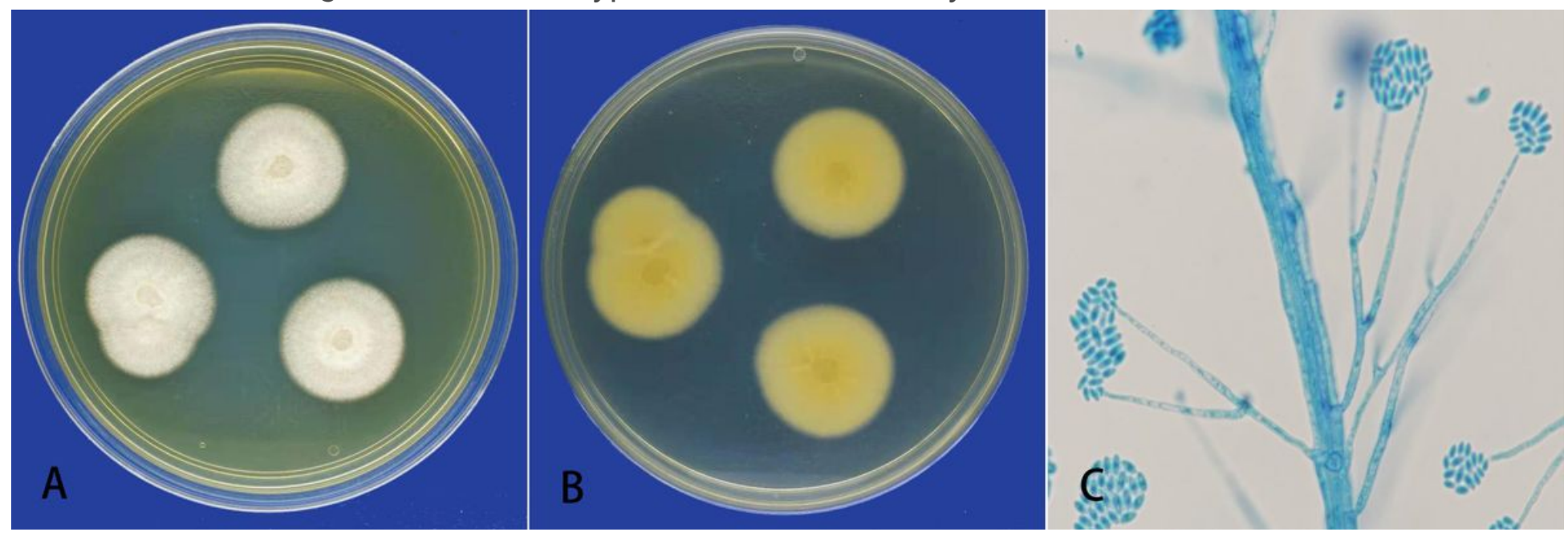

\section{Figure 4}

A Colony morphology of SDA medium cultured at $28^{\circ} \mathrm{C}$ for 8 days showed that the colonies grew slowly and were white, powdery, or fluffy, and B the reverse was light orange. C SDA medium was cultured at $28^{\circ} \mathrm{C}$ for 8 days and stained with lactophenol cotton blue staining (a magnification of $\times 400$ ). 